\title{
PRODUÇÃO DE BIODIESEL A PARTIR DA GORDURA DE FRANGO PELO MÉTODO DE TRANSESTERIFICAÇÃO HETEROGÊNEA
}

\author{
J. P. C. dos Santos, S. M. Viana e M. N. Sousa
}

Universidade de São Paulo, Escola de Engenharia de Lorena, Departamento de Engenharia Química

E-mail para contato: jp_lenos@ hotmail.com

\begin{abstract}
RESUMO - Este trabalho visa o estudo da produção de biodiesel a partir da reação de transesterificação da gordura de frango - gordura a qual é extremamente barata por ser extraída dos resíduos do abate da ave - catalisada por óxido de cálcio obtido de cascas de ovos. Através das técnicas de caracterização por Difratometria de Raios-X (DRX) e Micrografia Eletrônica de Varredura (MEV) foi possível caracterizar o catalisador comprovando a formação de óxido de cálcio a partir da casca de ovos após calcinação a $800{ }^{\circ} \mathrm{C}$ por $4 \mathrm{~h}$ e ainda revelar a superfície, sua morfologia e topografia. $\mathrm{O}$ método de extração da gordura de frango foi eficaz na obtenção de uma gordura límpida, homogênea e com baixo teor de ácidos graxos livres. Nas condições de reação, a conversão na transesterificação da gordura utilizando etanol foi menor do que naquelas que utilizaram o metanol. Para a análise de formação dos ésteres etílicos, bem como para a quantificação dos ácidos graxos insaturados presentes na gordura de frango, empregou-se ressonância magnética de hidrogênio $\left(\mathrm{RMN}-{ }^{1} \mathrm{H}\right)$.
\end{abstract}

\section{INTRODUÇÃO}

Combustíveis para motores diesel são usados em muitos setores e tem grande importância para a economia dos países. Sendo assim, o interesse no uso de combustíveis alternativos para esses motores aumentou como consequência da diminuição das reservas de petróleo e do aumento da consciência ambiental. Logo, o combustível que substituirá completa ou parcialmente o diesel de petróleo deve ser ambientalmente, economicamente e tecnicamente aceitável. Uma das várias opções de combustíveis alternativos para motores a diesel é o biodiesel, que pode ser produzido a partir de óleos vegetais ou gorduras animais; ele é um combustível não tóxico, biodegradável, que contém poucas quantidades de enxofre e não contribui para o efeito estufa, sendo assim, ambientalmente amigável.

Em geral, o biodiesel é produzido a partir de óleos vegetais de nível alimentício de alta qualidade e, por isso, possui um alto custo para comercialização, o qual é, justamente, a principal desvantagem desse combustível renovável, visto que, 70-95\% do custo de produção resulta do uso desses óleos. Dados do boletim mensal da Agência Nacional do Petróleo, Gás Natural e Bicombustível (ANP) mostram que, em maio de 2013, 78,43\% do biodiesel brasileiro foi produzido a partir do óleo de soja. Portanto, para tornar a produção do biodiesel 
mais atrativa para o comércio, matérias primas de baixo custo, tais como óleos de fritura e gordura animal, devem ser pesquisadas.

Muitos pesquisadores têm investigado a possibilidade do uso de gorduras animais na produção de biodiesel, no entanto, poucos optam pela gordura de frango como matéria prima de baixo custo para sua produção. No abate destas aves, os resíduos gerados têm alto teor de gordura, cujo destino vem a ser objeto de discussão, já que a utilização de subprodutos de origem animal, como carne de frango, na alimentação de ruminantes ou produção de ração animal (bovinos, bubalinos, caprinos e ovinos) foi proibida (Instrução Normativa $\mathrm{n}^{\circ} 41$, 08/10/2009). Devido a essa proibição, desprovidos de destino, os resíduos resultantes do abate de frango se tornam um poluente potencial. No abate de aves cerca de $30 \%$ do peso da ave viva é rejeitado na forma de resíduos, compostos por sangue, penas, vísceras e ossos, dos quais se obtém cerca de $11 \%$ de gordura (Gomes et al., 2004).

As desvantagens no uso da gordura de frango para a produção do biodiesel dizem respeito à presença de ácidos graxos livres (AGL) e água, em grandes proporções. Para um alto rendimento na formação de ésteres foi sugerido que o teor de ácido deva ser menor do que cinco miligramas por grama de óleo, e a presença de água deve estar abaixo de 0,3\%. Entretanto, ainda que haja alta concentração de AGL e água no óleo utilizado, a produção de biodiesel é possível através da inclusão de etapas de pré-tratamento no processo. O método mais eficaz observado por pesquisadores é a utilização de catálise ácida para esterificar os AGL a ésteres e, assim, diminuir a acidez da matéria prima, sem comprometer o rendimento final da reação. Mesmo necessitando de pré-tratamento para a obtenção de altos rendimentos em ésteres, o baixo custo e a possibilidade de transformar esse resíduo poluente em matéria prima para produção de biocombustíveis fazem da gordura de frango importante objeto de estudo.

Tratando-se da produção de biodiesel, o método industrial mais amplamente utilizado é um processo de transesterificação catalisada por base, a qual utiliza hidróxido de potássio $(\mathrm{KOH})$ ou hidróxido de sódio $(\mathrm{NaOH})$ como o catalisador, e metanol $(\mathrm{MeOH})$ como o álcool inferior. A vantagem deste processo é a produção de ésteres metílicos em rendimento muito alto, sob condições brandas e com tempo de reação de aproximadamente uma hora (Meher et al., 2006). No entanto, há vários problemas relacionados a esse processo, dentre eles, a dificuldade para lidar com o sabão que pode se formar dependendo do tipo de óleo usado, dificultando, assim, a separação do produto (Kwiecien et al., 2009). Os custos de produção através desse método são bastante elevados (Ma e Hanna, 1999), já que, para atender os critérios de qualidade estipulados pelas normas internacionais,o processo precisa envolver várias etapas de lavagem e purificação devido à dificuldade de remoção dos vestígios de potássio e sódio remanescentes no produto e a existência de desafios técnicos para a separação da glicerina. Além disso, o custo resultante da maior quantidade de água empregada nas lavagens e do consequente tratamento do efluente é elevado. Logo, para diminuir etapas de separação e reduzir o custo da produção de biodiesel, um grande número de métodos alternativos foi desenvolvido; dentre eles, o processo supercrítico (Minami e Saka, 2006), o processo enzimático (Shimada et al., 2002) e o processo por catálise heterogênea, que será o método utilizado neste trabalho.

No presente trabalho estudou-se a transesterificação da gordura de frango, um resíduo disponível no mercado e com propriedades que possibilitam a produção do biodiesel, utilizando óxido de cálcio $(\mathrm{CaO})$ obtido pela calcinação de cascas de ovos. 


\section{METODOLOGIA EXPERIMENTAL}

\subsection{Preparação do Catalisador}

Catalisadores de óxido de cálcio foram obtidos a partir de cascas de ovos. As cascas de ovos foram lavadas com água destilada até a remoção de toda parte orgânica que fica aderida à casca. Após a lavagem, as cascas foram trituradas com o auxilio de almofariz e pistilo para, então, serem pesadas e colocadas na mufla para o processo de calcinação. A calcinação das cascas foi feita a $800{ }^{\circ} \mathrm{C}$ por um período de 4 horas. Após a calcinação, as cascas de ovos foram pesadas, armazenadas em frascos e colocadas no dessecador.

\subsection{Caracterização do Catalisador}

A análise granulométrica do catalisador foi realizada empregando um conjunto de peneiras Tyler nos tamanhos 20, 28, 35, 42, 48 e 65 mesh.

O ensaio de basicidade utiliza indicadores de Hammet e tem o objetivo de evidenciar a formação do óxido de cálcio na casca de ovo calcinada, comparando a força básica da casca de ovo antes e após a calcinação. Neste trabalho os indicadores usados estão mostrados na Tabela 1. O ensaio ocorreu através do gotejamento do indicador sobre as amostras de casca de ovo, com posterior observação de mudança de cor (viragem).

Tabela 1 - Propriedades dos indicadores utilizados no ensaio de basicidade

\begin{tabular}{|c|c|c|c|}
\hline \multirow{2}{*}{ Indicador } & \multicolumn{2}{|c|}{ Cor } & \multirow{2}{*}{$\begin{array}{c}\text { Zona de viragem } \\
\text { (pH) }\end{array}$} \\
\cline { 2 - 4 } & Ácido & Básico & $8,2-10,0$ \\
\hline Fenolftaleína & Incolor & Roxa & $9,4-10,6$ \\
\hline Timolftaleína & Incolor & Azul & $10,0-12,0$ \\
\hline $\begin{array}{c}\text { Amarelo de } \\
\text { Alizarina }\end{array}$ & Amarelo & Vermelho & 1 \\
\hline
\end{tabular}

A análise por Difratometria de Raios-X (DRX) permite identificar as fases cristalinas em sólidos e neste trabalho foi empregada a técnica do pó, em um aparelho marca Seifert, modelo Isso-Debyeflex 1001, com radiação $\mathrm{CuK} \alpha$ com filtro de Ni. A tensão e a corrente utilizadas nos experimentos foram $40 \mathrm{kV}$ e $30 \mathrm{~mA}$, respectivamente. Os ângulos de Bragg (20) foram varridos entre 10 e 90 graus, com passo angular de 0,005 e tempo de contagem de 3 s por ponto.

A análise por Microscopia Eletrônica de Varredura (MEV) foi realizada para a obtenção das informações sobre a superfície dos sólidos quanto à morfologia e topografia. Para tanto, as amostras tiveram recobrimento de ouro e foram analisadas num equipamento da Hitachi, modelo TM 3000 com ampliações de 500, 1000, 2000 e 3000 vezes. 


\subsection{Extração, Análise e Pré Tratamento da Gordura de Frango}

A gordura de frango foi obtida a partir de resíduos do corte de frango de um açougue. Através do cozimento dos resíduos com adição de água, houve a formação de uma camada de gordura, a qual foi separada por decantação. Após a separação de fases, a gordura passou por filtração para remoção dos sólidos suspensos, com posterior lavagem utilizando água quente. Após a lavagem, a gordura foi novamente separada das outras fases, armazenada em frascos e mantida sob refrigeração para ser empregada nas reações de transesterificação.

Para avaliar a qualidade da gordura de frango extraída foram feitas análise de índice de acidez por titulação com solução aquosa de hidróxido de potássio $(1 \mathrm{~g} / \mathrm{L})$ e quantificação dos ácidos graxos insaturados pela técnica de $\mathrm{RMN}-{ }^{1} \mathrm{H}$.

Em alguns experimentos foi realizado um pré-tratamento da gordura para a redução dos ácidos graxos livres. Para isso, adicionou-se $30 \% \mathrm{~m} / \mathrm{m}$ de etanol e $8 \% \mathrm{~m} / \mathrm{m}$ de ácido sulfúrico, em relação à quantidade da gordura de frango, sob agitação a $60^{\circ} \mathrm{C}$ por 2 horas. Em seguida, a gordura foi lavada com água quente e centrifugada.

\subsection{Reação de Transesterificação e Planejamento de Experimentos}

A reação de transesterificação da gordura de frango foi realizada em um balão de fundo redondo, de $250 \mathrm{~mL}$, acoplado a um condensador e agitação magnética e a mistura reacional foi aquecida até a temperatura de reação desejada através de banho-maria. Após o tempo de reação programado, o catalisador foi separado do produto através de filtração e a mistura final foi colocada em um funil de separação, onde ocorreu a separação das fases contendo o biodiesel (ésteres) e o glicerol. Após a separação, os ésteres de alquila foram lavados com água destilada quente e levados para um evaporador rotativo para a remoção do álcool residual e o produto foi transferido para um frasco âmbar contendo sulfato de sódio anidro para remoção de traços de água, durante uma noite. Em seguida, a amostra foi centrifugada para a análise por ressonância magnética nuclear de hidrogênio $\left(\mathrm{RMN}-\mathrm{H}^{1}\right)$.

Buscando otimizar as condições da reação de transesterificação, utilizou-se o método de Plackett-Burman, pelo qual oito experimentos foram executados. As variáveis estudadas foram: temperatura de reação $(\mathrm{T})$, razão molar etanol/gordura (RM), concentração do catalisador $(\mathrm{c})$, pré tratamento $(\mathrm{PT})$ e tempo de reação $(\mathrm{t})$; cujas condições experimentais são mostradas na Tabela 2.

Tabela 2 - Limites superior e inferior das variáveis estudadas

\begin{tabular}{|c|c|c|}
\hline Variável & Limite inferior & Limite Superior \\
\hline T $\left({ }^{\circ} \mathbf{C}\right)$ & 60 & 75 \\
\hline RM & $15: 1$ & $25: 1$ \\
\hline $\mathbf{c}(\boldsymbol{\%} \mathbf{m} / \mathbf{m})$ & 5 & 10 \\
\hline PT & Sem & Com \\
\hline t $(\mathbf{h})$ & 2 & 4 \\
\hline
\end{tabular}




\section{RESULTADOS E CONCLUSÕES}

Durante a calcinação ocorre a formação do óxido de cálcio e liberação do dióxido de carbono, conforme Equação 1, cujo valor teórico de perda de massa é de 44\%. Os resultados da análise granulométrica, apresentados na Tabela 3, mostraram que houve redução no tamanho das partículas de sólido após a calcinação, assim como a esperada redução na massa das cascas de ovos, que foi de $46,68 \%$.

$$
\mathrm{CaCO}_{3} \rightarrow \mathrm{CaO}+\mathrm{CO}_{2} \quad \text { (Equação 1) }
$$

Tabela 3- Análise granulométrica empregando conjunto de peneiras

\begin{tabular}{|c|c|c|c|}
\hline Tyler & mm & \multicolumn{2}{|c|}{ \% massa } \\
\hline & & Casca de ovo & Casca de ovo calcinada \\
\hline 20 & 0,850 & 3,96 & 0,55 \\
\hline 28 & 0,600 & 43,40 & 24,54 \\
\hline 35 & 0,420 & 28,12 & 33,01 \\
\hline 42 & 0,355 & 8,43 & 9,51 \\
\hline 48 & 0,300 & 3,87 & 5,07 \\
\hline 65 & 0,212 & 3,02 & 4,39 \\
\hline Fundo & fundo & 8,71 & 22,92 \\
\hline & Massa (g) & 56,00 & 29,86 \\
\hline
\end{tabular}

Os ensaios de basicidade indicaram que a calcinação levou à mudança na basicidade da amostra, conforme pode ser observado na Figura 1 onde o indicador utilizado foi a timolftaleína. Observou-se que na casca de ovo in natura não há viragem da cor e após a calcinação ocorre mudança da cor em todos os indicadores testados.

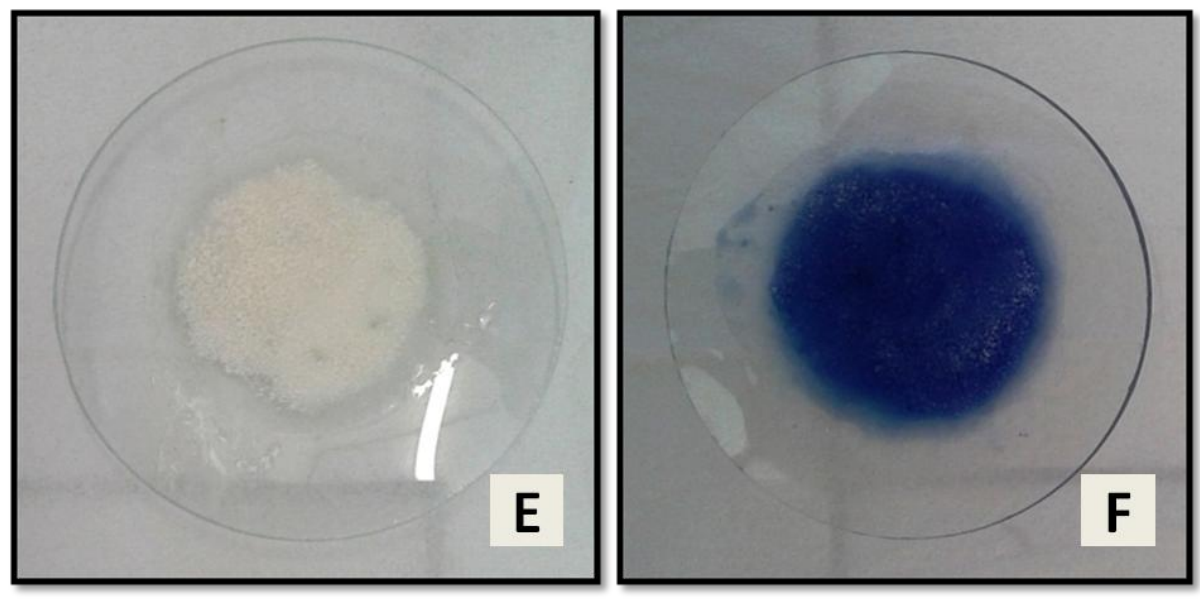

Figura 1- Ensaio com o indicador timolftaleína, casca de ovo in natura $(\mathrm{E})$ e casca de ovo calcinada $(\mathrm{F})$. 
As análises de DRX das amostras são apresentadas nas Figuras 2 e 3. Pode ser observadas as fases cristalinas de carbonato de cálcio na casca de ovo in natura e de óxido de cálcio na casa de ovo calcinada, por comparação com as fichas cristalográficas de padrões de $\mathrm{CaCO}_{3}$ e $\mathrm{CaO}$.

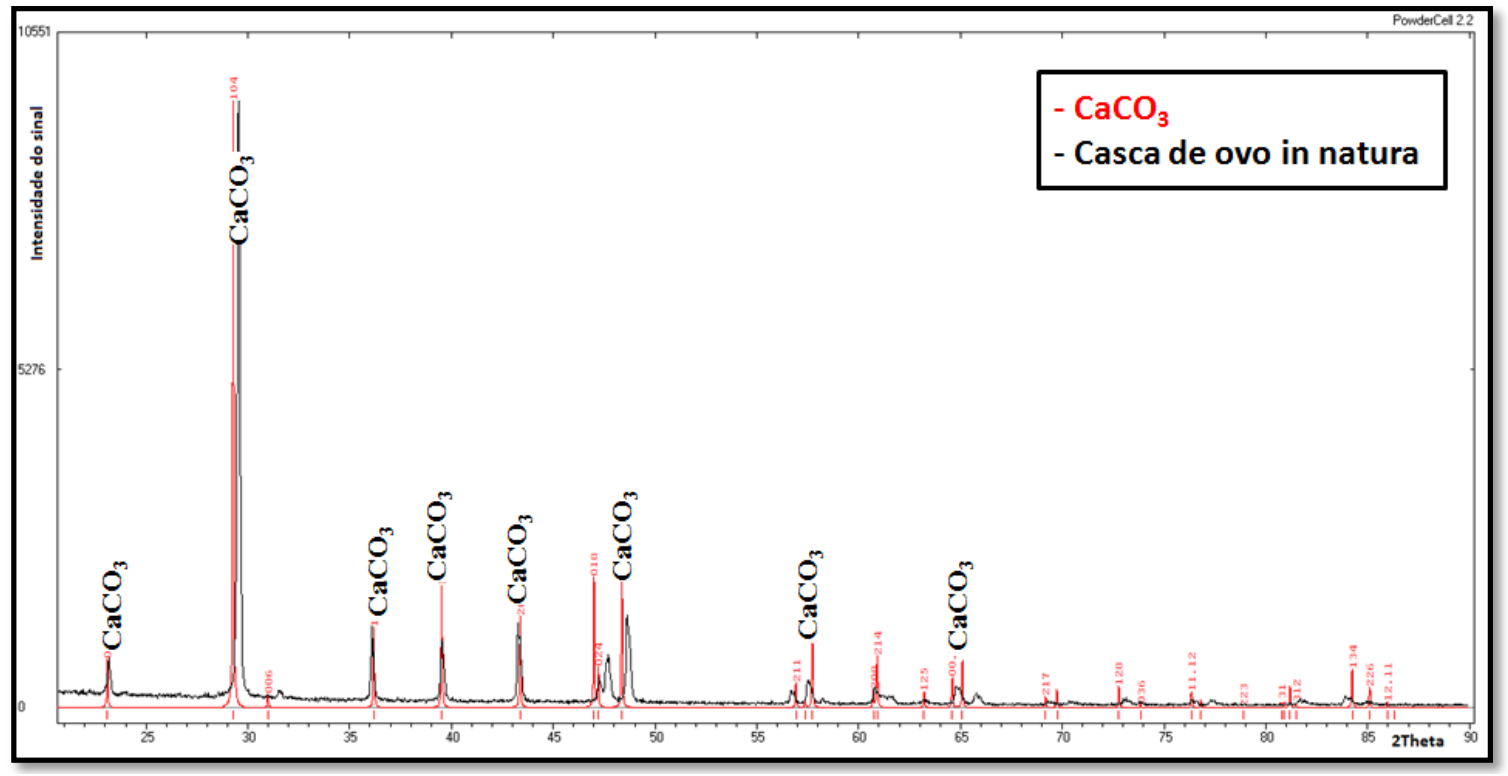

Figura 2 - Difratogramas da casca do ovo in natura (preto) e do padrão $\mathrm{CaCO}_{3}$ (vermelho).

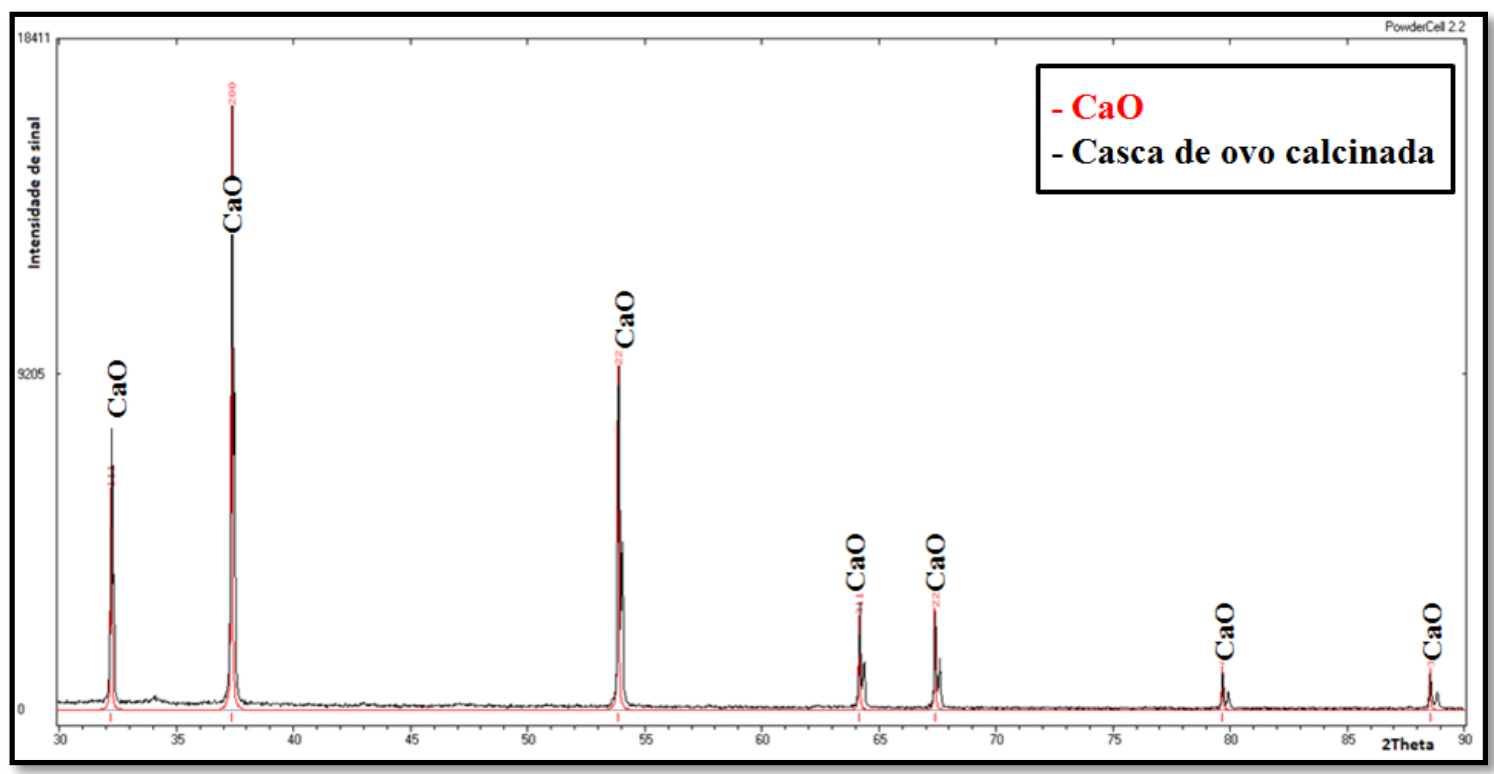

Figura 3 -Difratogramas da casca de ovo calcinada (preto) e do padrão $\mathrm{CaO}$ (vermelho).

Das análises por microscopia eletrônica de varredura (MEV), Figura 4, observa-se a mudança na superfície das amostras após a calcinação, notando-se a formação de pequenas partículas esféricas. A forma e o tamanho das partículas de óxido de cálcio na ordem de $30 \mu \mathrm{m}$ 
e de forma esférica, segundo Navajas et al. (2013), não leva a um bom rendimento nas reações de transesterificação.

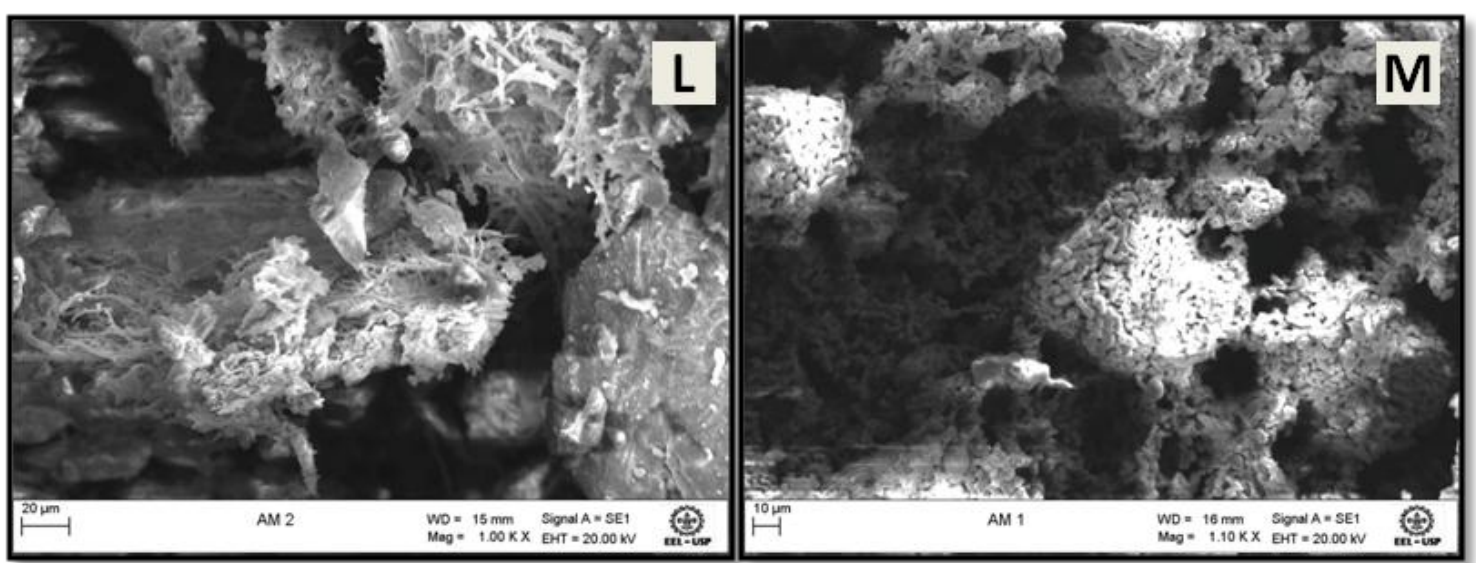

Figura 4 Micrografias por MEV(1000x): casca do ovo in natura (L), casca calcinada (M).

O método adotado de extração da gordura de frango foi adequado pois, a partir do cozimento dos resíduos de cortes de frango com água, filtração e lavagens, obteve-se uma gordura homogênea, livre de partículas sólidas. O índice de acidez foi igual a 4,97 mg KOH/g e o teor de ácido graxo livre igual a 2,5\%, adequados para a produção de biodiesel (Figura 5).

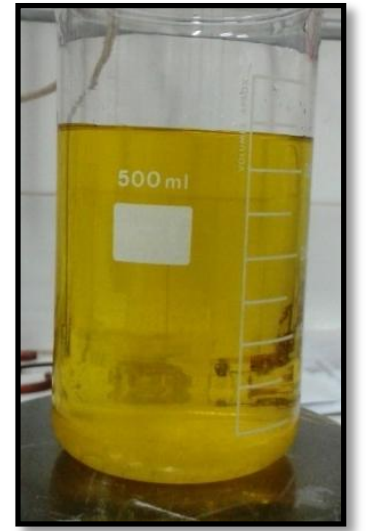

Figura 5 - Gordura de frango extraída.

A técnica de $\mathrm{RMN}-{ }^{1} \mathrm{H}$ foi empregada conforme metodologia publicada por Garcia (2006) para caracterizar a gordura em relação ao teor de ácidos graxos insaturados. Na Tabela 4 é apresentada a constituição da gordura de frango extraída, a predominância do ácido graxo 18:1 está de acordo com o trabalho de Boey et al.(2009) que estudaram a composição da gordura de frango sob as variáveis da forma de criação, de alimentação e da linhagem dos frangos.

Os produtos das reações de transesterificação da gordura de frango e etanol, após tratamento para separação da fase orgânica, foram analisados por $\mathrm{RMN}-\mathrm{H}^{1}$ empregando a metodologia proposta por Garcia (2006). O catalisador obtido da calcinação a $800^{\circ} \mathrm{C}$ de casca de ovos não apresentou atividade catalítica nas condições testadas. 
Tabela 4- Teor de ácidos graxos insaturados constituintes da gordura de frango

\begin{tabular}{|c|c|}
\hline $\begin{array}{c}\text { Ácidos graxos } \\
\text { Insaturados (AGI) }\end{array}$ & $\begin{array}{c}\text { Porcentagens de AGI da gordura do frango } \\
(\%)\end{array}$ \\
\hline $\mathbf{1 8 : 1}$ & 61,8 \\
\hline $\mathbf{1 8 : 2}$ & 24,3 \\
\hline $\mathbf{1 8 : 3}$ & 0,2 \\
\hline
\end{tabular}

Conclui-se que o óxido de cálcio produzido por este processo não foi ativo, fato o qual pode ser atribuído, à sua morfologia, como sugerido no estudo de Navajas et al.(2013). Entretanto, experimentos extras, empregando metanol alcançaram conversões de até $13 \%$.

\section{REFERÊNCIAS}

ANP - Agência Nacional do Petróleo, Gás Natural e Biocombustíveis : http://www.anp.gov.br, Boletim Fevereiro de 2013.

BOEY, P.; MANIAM, G.; HAMID, S. Biodiesel production via transesterification of palm olein using waste mud crab (Scylla serrata) shell as a heterogeneous catalyst. Bioresour. Technol. n. 100, p. 6362-6368, 2009.

GARCIA,C. M. Transesterificação de óleos vegetais. Dissertação de mestrado Universidade Estaduas de Campinas, Campinas, São Paulo, Brasil, 2006.

GOMES, L.; SOUZA, S.; BARICCATTI, R.; SOUZA, J. Potencial de produção de biodiesel a partir do óleo de frango nas cooperativas do oeste do Paraná. Varia Scientia (4), 133-146. 2004.

KWIECIEN, J.; HAJEK, M.; SKOPAL, F. The effect of the acidity of rapeseed oil on its transesterification. Bioresour. Technol. p. 5555-5559, 2009.

MA, F.; HANNA, M. A. . Biodiesel production: a review. Bioresour. Technol. n. 70, p. $1-15,1999$.

MEHER, L. C.; SAGAR, D. V.; NAIK, S. N. Technical aspects of biodiesel production by transesterification-a review. Renew Sustain Energy Rev, v.10, p. 248-268, 2006.

MINAMI, E.; SAKA, S. Kinetics of hydrolysis and methyl esterification for biodiesel production in two-step supercritical methanol process. Fuel 85. p. 2479-2483, 2006.

NAVAJAS, A.; ISSARIYAKUL, T.; ARZAMENDI, G.; GANDÍA, L. M.; DALAI, A. K. Development of eggshell derived catalyst for transesterification of used cooking oil for biodiesel production. Asia-Pacific Journal of Chemical Engineering , p. 742-748, 2013.

SCHMAL, M. Catálise Heterogênea, Rio de Janeiro, Synergia Editora, 2011

SHIMADA, Y.; WATANABE, Y.; SUGIHARA, A.; TOMINAGA, Y. Enzymatic alcoholysis for biodiesel fuel production and application of the reaction to oil processing. $J$. Mol. Catal. B: Enzym.v. 17,p. 133-142, 2002. 